\title{
Rate of Contaminated Blood Cultures (CBCs) at Tertiary Healthcare Setting, Saudi Arabia
}

\section{Lina Sallam*, N Kaabia, A Al Aidaroos and A Al Odayani}

Infection Control Department, Prince Sultan Military Medical City, Riyadh, Saudi Arabia

*Corresponding Author: Lina Sallam, Infection Control Department, Prince Sultan Military Medical City, Riyadh, Saudi Arabia.
Received: February 25, 2021

Published: March 30, 2021

(C) All rights are reserved by Lina Sallam., et al.

\begin{abstract}
Contaminated blood cultures (CBCs) leads to an avoidable antibiotic treatment and inappropriate diagnosis. It is a critical and frequent problematic seen in diagnostic laboratory which affects the quality of service and intimidates the patient's safety. To insure the quality of the medical services provided by our institution, the aims of this study were: i) to determine the rate of contamination among blood cultures collected from all patients in our hospital, and ii) to determine the clinical areas and categories of patients with high rate of CBCs. This is an observational study conducted at PSMMC, for 6 months (February-July 2019). CBCs were defined according to contamination criteria provided by College of American Pathologists (CAP). Patients' microbiological and demographical data were collected from electronical medical records. Among 786 positive blood cultures collected during the study period, 41 (5\%) samples were excluded due to deficient data to meet CAP criteria, 113 (15.1\%) were recognized as CBCs, 73\% from the latest were drawn from adult. Detected organisms among these contaminants were $56.6 \%$ Coagulase-negative Staphylococcus CoNS; $16.8 \%$ Corynebacterium spp; $16.8 \%$ Streptococcus viridians; and 8.8\% Bacillus. Remarkably, 28\% of CBCs were collected from patients when treated in the emergency rooms ER, 16\% were noticed in the acute medical unit, while 19\% of samples were taken from outpatients departments OPD. To conclude, this study presented a high rate of CBCs collected from ER and OPD, however, a quality improvement project, include all stakeholders, is currently ongoing in order to reduce the rate of CBCs.
\end{abstract}

Keywords: Contaminated Blood Cultures; Streptococcus viridians; Corynebacterium spp; Bacillus

\section{Abbreviation}

CBCs: Contaminated Blood Cultures; CAP: College of American Pathologists; OPD: Outpatients Departments; CoNS: Coagulasenegative Staphylococcus; ER: Emergency Room; FPC: False Positive Culture; TNC: Truly Negative Culture; ASM: American Society for Microbiology; CLSI: Clinical Laboratory Standards Institute; PSMMC: Prince Sultan Military Medical City.

\section{Introduction}

In hospitalized patients, bloodstream infection remains one of the principal reasons leading to mortality among worldwide hos- pitals [1]. Sepsis and bacteremia are the most common associated illness to this infection [2]. Because of the worldwide bacteremiarelated high mortality, it is important to take every measure to reduce its prevalence and incidence to start effective empirical treatment as earliest as possible [3-6]. To ensure the quality of medical services provided by our institution, the aims of this study were to determine the rate of contamination among blood cultures collected from all patients in our hospital and to determine the clinical areas and categories of patients with high rate of CBCs. 
Contaminated blood cultures CBCs, also known as false positive samples, lead to an avoidable antibiotic's treatments and inappropriate diagnosis. It is a critical and frequent problem seen in diagnostic laboratory which affects the quality of service and intimidates the patient's safety. CBCs were defined according to contamination criteria provided by College of American Pathologists (CAP) [1]. False positive culture (FPC) compare to truly negative culture (TNC) increases the use of broad-spectrum antimicrobial drugs and prolong the patient stay in the hospital $[1,3,7,8]$. Economic impact of blood contamination was also mentioned, which might extend the length of hospitalization due to wrong diagnosis, laboratory tubes consumption, and staff overwhelming. Generally reported contamination rate in hospital is fluctuating from $0.6 \%$ to $12.5 \%$ world widely, while the highest rate is associated with emergency department settings $[2,4,6,9]$. However, one study reported that contamination rate in pediatric outpatients was $26 \%$ [2]. This false positive result misleads the diagnosis and can lead to unnecessary treatment $[2,9]$. According to the American Society for Microbiology (ASM) and Clinical Laboratory Standards Institute (CLSI), the rate of contaminated blood culture should not exceed $3 \%[2,3]$. The main goal of this research was to collect data from a daily microbiology report that was sent by the central laboratory to the infection control department at PSMMC. The rate of contamination was compared to an international benchmark that helps in measuring and evaluating quality and defect of the service in each clinical area. This will contribute on how to improve the quality of the particular health services.

\section{Methods}

This is an observational study conducted at Prince Sultan Military Medical City (PSMMC), for 6 months (February-July 2019). Sample size is approximately 800 positive blood cultures generated from laboratory report, out of this number; 197 blood samples met the contamination criteria. CBCs Criteria are listed as following: when one of the following organisms present in $\leq 50 \%$ of all blood cultures sets ( 2 bottles) that obtained from one patient on the same day (Coagulase-negative Staphylococcus CoNS, Corynebacterium spp, Streptococcus viridians, Micrococcus spp, and Propionibacterium spp). Patients' microbiological and demographical data were collected from electronical medical records. Blood samples that drown in one bottle for culture did not meet the CAP criteria for contamination, and therefore were excluded. The rate of blood culture contamination was calculated by dividing the total number of contaminated blood cultures by the total number of blood cultures collected during the study period. We also determined rate of $\mathrm{CBC}$ s from all positive $\mathrm{BC}$, by dividing number of contaminated blood culture by total number of positive BC.

- Definition of contamination: Organisms that are not actually present in blood sample are growing in culture. Specific organism was identified to show false positive result. Sample has to meet CAP criteria of false positive blood culture or contaminated blood culture when precise organisms present in $\leq 50 \%$ of all blood cultures sets, 2 bottles, that obtained from one patient on the same day; Coagulase-negative Staphylococcus (CoNS). Corynebacterium spp, Streptococcus viridians, Micrococcus spp, Propionibacterium spp. Blood samples that drown in one bottle for culture does not meet the CAP criteria for contamination and therefore will be excluded.

- Calculation of the contamination rate: The rate of blood culture contamination was calculated by dividing the total number of contaminated blood cultures by the total number of blood cultures collected during the study period. We also determined the rate of $\mathrm{CBC}$ s from all positive $\mathrm{BC}$, by dividing number of contaminated blood culture by total number of positive BC.

- Statistical analysis: All data will be analyzed using descriptive and inferential statistic via IBM SPSS version 20. Descriptive statistics, such as median or mean, was analyzed based on the normality of the data. Moreover, continuous variables such as, age, date of admission and collection were calculated as duration (days). Categorical variable such as, gender, patient location, origin of infection, specimen type, site of the specimen, also the type of organisms is presented as frequencies $(\mathrm{N})$ and percentages $(\%)$.

- Patients' confidentiality: To ensure confidentiality, all patients' identities were kept anonymous.

\section{Results}

According to our laboratory surveillance, the mean rate of contaminated blood culture, during the study period was $2.4 \%$. This rate was counted from hospital wide blood samples collected during the study period. However, out of 786 positive blood cultures, $113(14.3 \%)$ were recognized as CBCs, $73 \%$ from the latest was 
drawn from adult and $17 \%$ from pediatric, $(n=57) 50.5 \%$ was recognized as hospital onset and $(n=56) 49.5 \%$ was community onset, however, $(\mathrm{n}=41) 5 \%$ samples were excluded due to deficient data to meet CAP criteria (Table 1). Relative to these results, the drawing site of $(n=94) 83 \%$ of CBCs were the peripheral veins, $(n=14) 12 \%$ from central line, and $(n=5) 4 \%$ were from arterial (Table 1). The most frequent microorganism isolated from CBCs was Coagulase-negative Staphylococcus (56.6\%) followed by (16\%) of Strep viridans and Corynebacterium, (8\%) by Bacillus spp, and $(0.8 \%)$ by Micrococcus spp. (Table 1, Figure 1). Remarkably, ( $n=32$ ) $28 \%$ of CBCs were collected from patients treated in the emergency rooms (ER), $(n=22) 19 \%$ from acute medical surgical unit, $(n=19) 16 \%$ were from outpatient clinics (Figure 2).

\section{Discussion}

Our central laboratory serves three other regional healthcare institutions in addition to PSMMC the rate of CBCs as reported during the same period of our study by the Lab was $2.4 \%$. This rate was calculated for all blood samples received from all served hospitals, and, it was difficult to extract the blood cultures only received by PSMMC patients. In addition, our original data excluded the positive duplicated samples, as we were monitoring the positive blood cultures. Accordingly, it was a challenge to specify the rate of CBCs for PSMMC patients only. in this study, CBCs was calculated among all positive blood samples and found to be $18 \%$. However, benchmarking our data $(2.4 \%)$ with local and international data shows an acceptable range of contamination $[1,2,9]$.

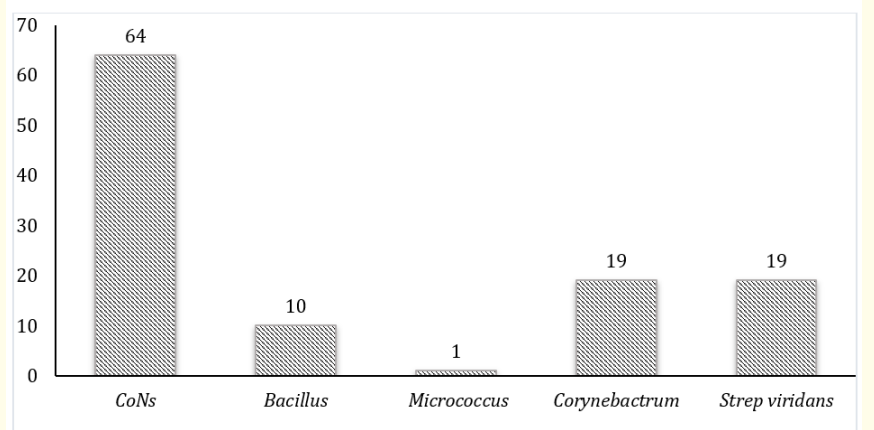

Figure 1: Types and numbers of isolated organisms from contaminated samples.

CoNS: Coagulase-negative Staphylococcus.

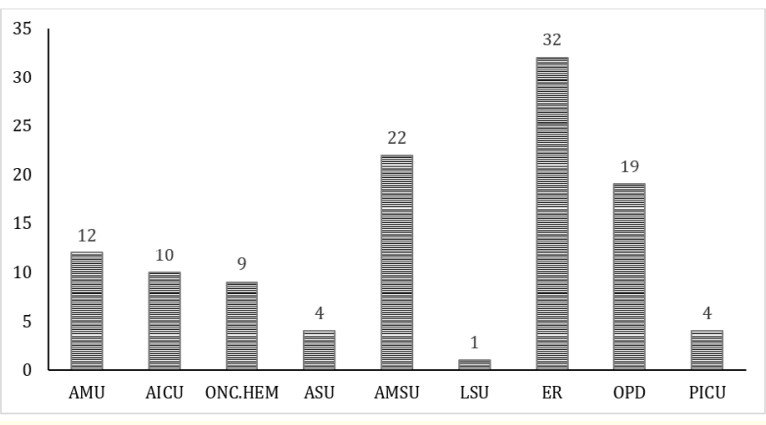

Figure 2: Distribution of Contaminated BCs among the Facilities of the Hospital.

AMU: Acute Medical Unit; AICU: Adult Intensive Care Unit; Ocn. Hem: Oncology/Hematology Unit; ASU: Acute Surgical Unit; AMSU; Acute Medical/ Surgical Unit; LSU: Long Stay Unit; ER: Emergency Room; OPD: Out patients department; PICU: Pediatric Intensive Care unit.

Published studies found that reducing the rate of CBCs is associated with decreased length of stay, subsequent hospitalization, and unnecessary antibiotic regimens $[1,2,9]$. For health care providers, CBCs was addressed as serious economic and quality issues as it led to unfavorably misdiagnosis and prolonged preventable hospitalization, which negatively disrupt the quality of care and raise the cost of service $[1,2,9,13,14]$. In a published study performed among children, researchers found that $26 \%$ of children with falsepositive cultures were primarily admitted to the hospital based on initial culture results that explain how CBCs cause false diagnosis and led to unnecessary hospitalization [9]. Other evident from several studies in the United States have reported the concern about an extensive cost associated with CBCs from adult patients $[1,9,10]$.

CoNS are definitely most often contaminants but have recently become significant microbial finding as an agent of bacteremia [1]. However, it is hard to determine whether both sets are presented without deep inspection and investigation, particularly when the present study's focus was based on microbiological reporting results without considering clinical features of the infected patients $[6,8,12]$. Therefore, clinical data, and patient characteristics must be considered and analyzed to differentiate between contaminated 
Table 1: Data of contaminated blood cultures from PSMMC patients.

\begin{tabular}{lccc}
\hline Data & Adult & Pediatric & Total \\
\hline Contaminated Blood Cultures & & & \\
CBC/PBC & $83 / 618(15.5 \%)$ & $30 / 127(30 \%)$ & $113 / 745(17 \%)$ \\
Origin of Contamination & & & \\
Community Onset & $34 / 83(40 \%)$ & $24 / 30(80 \%)$ & $58 / 113(51 \%)$ \\
Hospital Onset & $49 / 83(59 \%)$ & $8 / 30(26 \%)$ & $57 / 113(50 \%)$ \\
Types of Micro-Organisms & & & \\
CoNS & $47 / 83(56 \%)$ & $17 / 30(56 \%)$ & $64 / 113(56 \%)$ \\
Micrococcus spp. & $1 / 83(1 \%)$ & $0 / 30(0 \%)$ & $1 / 113(0.8 \%)$ \\
Bacillus spp. & $8 / 83(9.6 \%)$ & $2 / 30(6 \%)$ & $10 / 113(8 \%)$ \\
Corynebacterium spp. & $16 / 83(19 \%)$ & $3 / 30(10 \%)$ & $19 / 113(16 \%)$ \\
Strep Viridans & $9 / 83(10 \%)$ & $8 / 30(26 \%)$ & $19 / 113(16 \%)$ \\
Source of Contaminated Blood sample & & & \\
Peripheral & $71 / 83(85 \%)$ & $23 / 30(76 \%)$ & $94 / 113(83 \%)$ \\
Central Line & $10 / 83(12 \%)$ & $4 / 30(13 \%)$ & $14 / 113(12 \%)$ \\
Arterial & $2 / 83(2 \%)$ & $3 / 30(10 \%)$ & $5 / 113(4 \%)$ \\
\hline
\end{tabular}

CBC: Contaminated Blood Cultures; PBC: Positive Blood Cultures; CoNS: Coagulase-negative Staphylococcus.

culture and true infection of CoNS [11,12]. Adding to that point, there has been an increased percentage of CoNS bloodstream infection, this should be kept in mind especially in patients with intravascular-devices [1,12]. Careful and accurate evaluation of this isolate has to be monitored to avoid a consequent increase of antibiotic resistant patterns of Gram- positive cocci in healthcare facilities [12].

Relative to blood contaminant reduction approaches, many studies advocate the use of dedicated well-trained phlebotomy teams to collect blood cultures [1,10-12]. Hence, it would be an interesting to measure the rate of contamination after the implementation of the dedicated phlebotomist. This could reduce the rate and improve the quality of blood collection procedures if it could be applicable, this intervention could be addressed under an improvement project for a better quality of healthcare service.

In our study, the data collected from patients treated in ER, AMSU, and OPD showed the highest rate of CBCs. Thus, the elevated rate might be a result of the rapid practice by the non-phle- botomy staff in the emergency department (ED), as the nature of unpredictable critical situation and volume of patients in the ED is demanding a rapid response. A Study from The University of Michigan Health System (UMHS) Emergency Department (2013) has addressed that ED high volume of outpatients and crowding have adverse effect on the quality of blood culture and increase the number of CBCs, the study said, "ED crowding was associated with degraded performance of blood cultures" [13].

However, as this study was performed in a tertiary teaching hospital, it is common in such hospital for the contamination rate to exceed $6 \%$ or more [1]. Consequently, the results of the current study were expected, due to the huge number of medical and paramedical students and trainees, this is might explain, but not defend, the contaminants rate.

Finally, yet importantly, inappropriate aseptic techniques that practiced by staff is also a potential risk factor to increase the rate of CBCs, which needs a close observation to evaluate the proficient practice of blood draw for correct assessment on the accuracy of 
this procedure [1,14]. Studies have shown that several key factors may reduce the rate of $\mathrm{CBCs}$ during the blood drawn, for instance adhering to protocol, using of sterile gloves, cleaning tops of blood culture bottles with antiseptics and inoculating blood culture bottles before other blood tubes are all serious antiseptic performing that affect the accuracy of blood sampling [1,11,14].

\section{Conclusion}

A likely explanation for a tertiary teaching hospital is that to expect a high rate of blood contamination. This is an anticipated condition in such healthcare setting. Future study should emphasize on the technique applied when collecting blood samples. In addition, dedicated nurses in areas have had high contaminants rate might be an appropriate decision. Further study is needed to compare the current rate with the rate after the implementation of the development plane. In conclusion, better strategy in order to prevent contamination and improve the quality of care is the first action to be established.

\section{Bibliography}

1. Roth A., et al. "Reducing blood culture contamination by a simple informational intervention". Journal of Clinical Microbiology 48.12 (2010): 4552-4558.

2. Snyder Susan R., et al. "Effectiveness of practices to reduce blood culture contamination: a Laboratory Medicine Best Practices systematic review and meta-analysis". Clinical Biochemistry 45.13-14 (2012): 999-1011.

3. Wilson Michael L., et al. "Principles and procedures for blood cultures; approved guideline”. CLSI document M47-A. Clinical and Laboratory Standards Institute, Wayne, PA (2007).

4. Son JS., et al. "Bloodstream Infections and Clinical Significance of Healthcare-associated Bacteremia: A Multicenter Surveillance Study in Korean Hospitals". Journal of Korean Medical Sciences 25 (2010): 992-998.

5. Hayden R T., et al. "Diagnostic microbiology of the immunocompromised host". ASM Press (2009).

6. Viscoli Claudio. "Bloodstream Infections: The peak of the iceberg". Virulence 7.3 (2016): 248-251.

7. Safdar Nasia., et al. "Meta-analysis: methods for diagnosing intravascular device-related bloodstream infection". Annals of Internal Medicine 142.6 (2005): 451-466.

8. Lenz Ryan., et al. "The distinct category of healthcare associated bloodstream infections". BMC Infectious Diseases 12 (2012): 85.
9. Hall Keri K and Jason A Lyman. "Updated review of blood culture contamination". Clinical Microbiology Reviews 19.4 (2006): 788-802.

10. Gander Rita M., et al. "Impact of blood cultures drawn by phlebotomy on contamination rates and health care costs in a hospital emergency department". Journal of Clinical Microbiology 47.4 (2009): 1021-1024.

11. Bekeris Leonas G., et al. "Trends in blood culture contamination: a College of American Pathologists Q-Tracks study of 356 institutions". Archives of Pathology and Laboratory Medicine 129.10 (2005): 1222-1225.

12. Sidhu Shailpreet K., et al. "Significance of coagulase negative Staphylococcus from blood cultures: persisting problems and partial progress in resource constrained settings". Iranian Journal of Microbiology 8.6 (2016): 366-371.

13. Halverson Schuyler., et al. "Impact of hourly emergency department patient volume on blood culture contamination and diagnostic yield". Journal of Clinical Microbiology 51.6 (2013): 1721-1726.

14. Dawson S. "Blood culture contaminants". The Journal of Hospital Infection 87.1 (2014): 1-10.

\section{Assets from publication with us}

- Prompt Acknowledgement after receiving the article

- Thorough Double blinded peer review

- Rapid Publication

- Issue of Publication Certificate

- High visibility of your Published work

Website: www.actascientific.com/

Submit Article: www.actascientific.com/submission.php Email us: editor@actascientific.com

Contact us: +919182824667 